\title{
Glioblastoma extracellular vesicles: reservoirs of potential biomarkers
}

\author{
Jasmina S Redzic' \\ Timothy H Ung ${ }^{2}$ \\ Michael W Graner ${ }^{2}$ \\ 'Skaggs School of Pharmacy and \\ Pharmaceutical Sciences, ${ }^{2}$ Department \\ of Neurosurgery, School of Medicine, \\ University of Colorado Denver, \\ Aurora, CO, USA
}

This article was published in the following Dove Press journal:

Pharmacogenomics and Personalized Medicine

13 February 2014

Number of times this article has been viewed

\begin{abstract}
Glioblastoma multiforme (GBM) is the most frequent and most devastating of the primary central nervous system tumors, with few patients living beyond 2 years postdiagnosis. The damage caused by the disease and our treatments for the patients often leave them physically and cognitively debilitated. Generally, GBMs appear after very short clinical histories and are discovered by imaging (using magnetic resonance imaging [MRI]), and the diagnosis is validated by pathology, following surgical resection. The treatment response and diagnosis of tumor recurrence are also tracked by MRI, but there are numerous problems encountered with these monitoring modalities, such as ambiguous interpretation and forms of pseudoprogression. Diagnostic, prognostic, and predictive biomarkers would be an immense boon in following treatment schemes and in determining recurrence, which often requires an invasive intracranial biopsy to verify imaging data. Extracellular vesicles (EVs) are stable, membrane-enclosed, virussized particles released from either the cell surface or from endosomal pathways that lead to the systemic release of EVs into accessible biofluids, such as serum/plasma, urine, cerebrospinal fluid, and saliva. EVs carry a wide variety of proteins, nucleic acids, lipids, and other metabolites, with many common features but with enough individuality to be able to identify the cell of origin of the vesicles. These components, if properly interrogated, could allow for the identification of tumor-derived EVs in biofluids, indicating tumor progression, relapse, or treatment failure. That knowledge would allow clinicians to continue with treatment regimens that were actually effective or to change course if the therapies were failing. Here, we review the features of GBM $\mathrm{EVs}$, in terms of EV content and activities that may lead to the use of EVs as serially accessible biomarkers for diagnosis and treatment response in neuro-oncology.
\end{abstract}

Keywords: exosomes, microvesicles, brain tumors, proteomics, lipidomics, ribonomics

\section{Introduction}

Glioblastoma multiforme (GBM; World Health Organization [WHO] grade IV glioma) is the most common primary malignant brain tumor in the United States, striking some three in 100,000 people. Patients with GBM are faced with a poor prognosis as 5-year survival rates are less than $5 \%$ for all age groups (of those $\geq 75$ years of age, virtually none are alive by that time). ${ }^{1}$ Roughly 17,000 Americans are diagnosed with GBM each year, and the disease claims $\sim 13,000$ lives, with most succumbing in 1-2 years, despite gross total surgical resection, external beam radiation treatment, and the latest in multimodal chemotherapy. ${ }^{2}$ These therapies are relatively nonspecific and come at significant cognitive and physiopsychological costs to the patient, as the brain is an organ that can not tolerate much collateral damage. Despite the relatively short time course of the disease, the financial burdens at a familial level, are staggering, ${ }^{3}$ and at a 
national level, the therapies may represent the most expensive (ie, ineffective) treatments per life-year saved when factoring in quality-of-life adjustments. ${ }^{4,5}$ Thus, at the individual, familial, and a national level, GBMs take a horrendous toll.

\section{Glioblastoma: surgical and other therapeutic limitations}

GBMs are highly invasive malignant neoplasms of glial cells within the central nervous system that carry a very poor prognosis, despite advancements in surgical and oncological modalities. Neurosurgical resection of GBMs has failed to offer patients with a curative option as even radiological imaging with contrast enhancement of disease inadequately correlates with actual neoplastic disease burden (Figure 1A). Thus, gross total resection, using the radiological image of the apparent tumor margins, fails to address the microinfiltrative disease beyond the borders of the radiological depiction (which can be identified upon histological representation). ${ }^{6-8}$ Histologic characterization of GBMs commonly shows small areas of central necrosis, with a distinct pseudopalisading rim of anaplastic glial cells and a hyperplastic, hyperpermeable vasculature, which differentiates these tumors from lower-grade astrocytomas (Figure 1B and C). Examination of the pseudopalisading network of neoplastic glial cells is remarkable for disease heterogeneity. The neoplastic heterogeneity observed in GBM poses significant challenges in disease treatment as well-known oncological therapeutics fail to target the entire disease burden and may actually select for inherently resistant neoplastic cells. ${ }^{9-12}$ Further histological investigation of the brain parenchyma beyond the location of the radiographical depiction of the disease often reveals significant microinfiltrative tumor cells, which are thought to be a major contributor to the dismal prognosis of GBM. ${ }^{6,9}$ The inability to cure GBM via neurosurgical resection and the failure of our current therapeutics contribute to the urgent need for further GBM tumor characterization.

\section{Characterization of glioblastoma}

There have been remarkable strides made recently in the molecular genetic characterizations of GBMs, which have been valuable in the initial understanding of the detrimental effects of GBM and may prove useful in the stratification of patients by therapeutic response and/or in clinical trials. ${ }^{13}$ However, these types of tumor identifiers are obviously evident only after the tumor is present and accessible. For the purposes of serial monitoring during the course of treatment or the early detection of recurrent tumors, biofluid-based biomarkers will
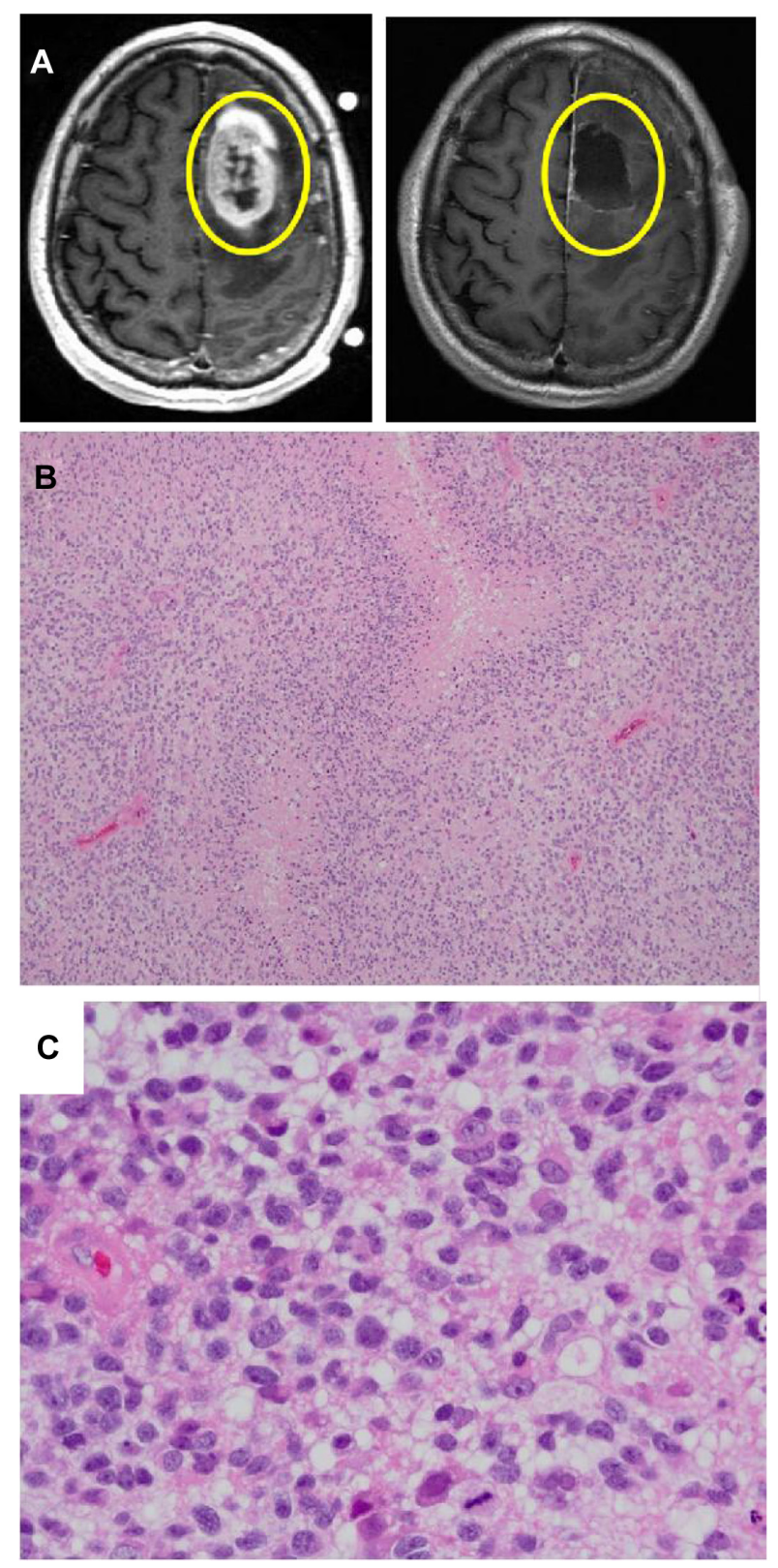

Figure I Radiographic (MRI) images and histology of GBMs.

Notes: (A) shows an MRI of a GBM before (left) and after (right) surgery. Yellow circles show the area of tumor (left) and the resection cavity following surgery (right). (B) (low power, 100x) and (C) (high power, 600x) are hematoxylin/eosin stains of a section of a GBM used in histopathologic diagnosis. (B) shows the typical hypercellularity, cytological atypia, and prominent pseudopalisading necrosis of a GBM. (C) at higher power (same tumor, different section), better illustrates the cellular atypia and mitotic activity in the GBM.

Abbreviations: GBM, glioblastoma multiforme; MRI, magnetic resonance imaging.

be essential tools. Virtually all biofluids (eg, serum/plasma, urine, cerebrospinal fluid, breast milk, semen, colostrum, bronchial lavage fluid, ascites fluid, saliva, and synovial fluid) have one set of common components - extracellular vesicles, consisting of exosomes and microvesicles. ${ }^{14,15}$

Extracellular vesicles (EVs) are membrane-enclosed, virus-sized $(30-1,000 \mathrm{~nm})$ nanovesicles that are released 
extracellularly from cells, either directly from the cell surface (called "microvesicles," "microparticles," or "ectosomes") by membrane "blebbing" or via the formation in an endocytic route, with fusion of a late endosome/multivesicular body with the plasma membrane ("exosomes") ${ }^{14,15}$ (Figure 2). Extracellular release deposits EVs into systemic or circulating fluids, with the potential for proximal and distal effects. These vesicles contain numerous cellular constituents, such as proteins, nucleic acids, lipids, and metabolites, that have common denominators in terms of the biogenesis of vesicle formation and cargo loading, but the constituents also have sufficient specificity that they can identify the cell of origin of their release. EVs are stable and protect the constituents from nucleases, proteases, and the other means of degradation found in the biologically harsh extracellular environments. ${ }^{16}$ EVs also have physicochemical properties that enable their purification via standard procedures. ${ }^{17,18}$ Based on these features, EVs appear to be veritable repositories of potential biomarkers for health and disease, ${ }^{19}$ and this is clearly evident in

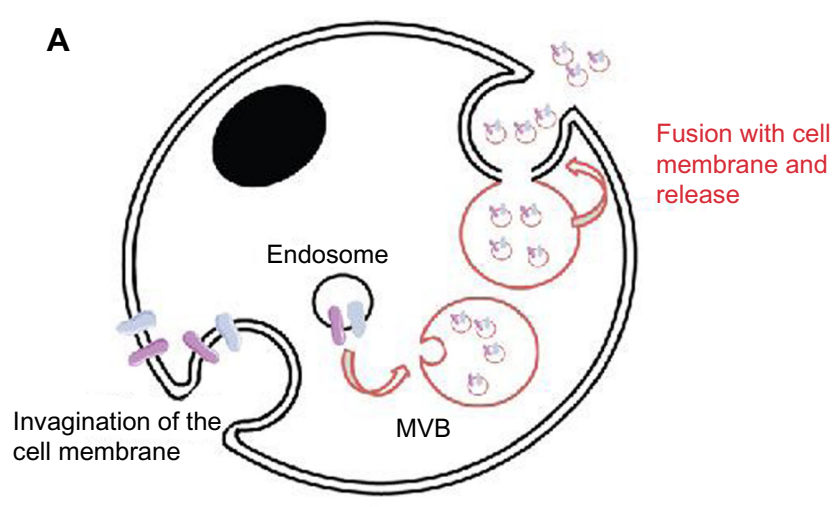

B

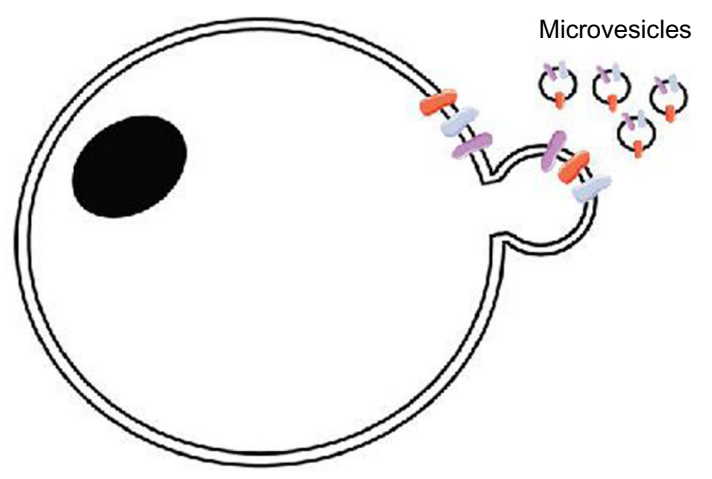

Figure 2 Two main modes of $\mathrm{EV}$ formation.

Notes: (A) shows the exosome pathway, whereby materials are taken from the cell surface into the endosomal system, with later invaginations forming MVB. If the MVB fuses with the plasma membrane, it releases the internal vesicles into the extracellular space as exosomes. (B) shows the formation of microvesicles as shed vesicles, budding directly off from the plasma membrane.

Abbreviations: $\mathrm{EV}$, extracellular vesicle; MVB, multivesicular body. oncology as well, ${ }^{20}$ especially in neuro-oncology, where the needs for diagnostic, prognostic, and predicative biomarkers are urgent. ${ }^{21}$

\section{Biomarkers - general considerations}

The term "biomarker" (as in "biological marker") seems straightforward, but some definitions are applicable. Typically a biomarker refers to "a characteristic that is objectively measured and evaluated as an indicator of normal biological processes, pathogenic processes, or pharmacologic responses to a therapeutic intervention." ${ }^{22}$ This is a broad statement but encompasses most aspects. There are three recognized subcategories of biomarkers: 1) diagnostic markers that identify the presence of and specific type of disease; 2) prognostic markers used to imply outcomes independently of therapy; and 3) predictive markers that can inform in the context of particular treatment response. For gliomas, we currently know of nothing truly useful as an early-stage diagnostic biomarker as most GBMs present as full-blown tumors with little or no prior clinical evidence of their existence..$^{23}$ There are certain molecular features of GBMs that aid in and verify diagnoses, and some of those are also prognostic and may be predictive (study of this is ongoing). ${ }^{24}$ The current line of thought in this area is that recurrent genetic abnormalities are able to subcategorize GBMs into groups, defined as Classical, Mesenychmal, Proneural, and Neural. ${ }^{25}$ The main features of these designations are shown in Table 1. The gene expression patterns and genomic alterations associated with the GBM categories may eventually lead to better therapeutic strategy design, but for now, the most disturbing correlation is that only patients with the Proneural subtype show any trend towards longer survival compared with patients in the other three categories. Nonetheless, that prolonged survival is not statistically significantly different compared with the other groups and in general, it is still less than 2 years postdiagnosis.

It is important to realize that the molecular signatures of GBMs are based on analyses of actual tumor specimens or biopsies from them. Thus, the use of those molecules in the ongoing monitoring of patient progress or response to therapies may be limited, suggesting that biofluid-based biomarkers are likely to be of far more practical benefit. Circulating biomarkers may be serially collected, which is essential for therapeutic assessment, and the (usually) minimally invasive nature of collection (compared with the substantial intracranial interventions at surgery or biopsy) is also attractive.

As mentioned above, most GBMs present acutely, with relatively few clear indications of the oncoming pathogenesis 
Table I Subclassifications of glioblastomas (based on TCGA) ${ }^{25}$

\begin{tabular}{|c|c|c|c|c|}
\hline Subclass & Chromosome alterations & Gene/protein expression changes & Drugs/targets & Relative surviva \\
\hline Classical & $\begin{array}{l}\text { cs7 gain with cs } 10 \text { loss; } \\
\text { loss } 9 p 21.3\end{array}$ & $\begin{array}{l}\downarrow \text { CDKN2A, PTEN } \\
\uparrow \text { NES NOTCH3 JAGI } \\
\text { LFNG SMO GASI GLII EGFR amplified; } \\
\text { EGFRvIII expression }\end{array}$ & $\begin{array}{l}\text { TMZ } \\
\text { MDM2 inhibitors }\end{array}$ & Poor \\
\hline Mesenchymal & Loss $|7 q| \mid .2$ & $\begin{array}{l}\uparrow \text { CHI3LI/YKL40 MET CD44 MERTK } \\
\uparrow T N F \text { and NFKB family } \\
\text { Mutated p53 PTEN NFI }\end{array}$ & $\begin{array}{l}\text { TMZ/XRT } \\
\text { Bevacizumab } \\
\text { VEGFR inhibitors }\end{array}$ & Poor \\
\hline Proneural & $\begin{array}{l}\text { Amplification cs7; loss cs } 10 \text {; } \\
\text { amplification } 4 q 12 \text {, but low } \\
\text { amplification of } 7 \text { p I I.2 }\end{array}$ & $\begin{array}{l}\uparrow \text { PDGFRA OLIG2 TCF3 NKX2-2 SOX } \\
\text { DCX DLL3 ASCLI TCF4 } \\
\text { Mutated IDHI P53 PIK3CA/PIK3RI }\end{array}$ & $\begin{array}{l}\text { Inhibitors of } \\
\text { HIF } \\
\text { PI3K } \\
\text { PDGRFA }\end{array}$ & $\begin{array}{l}\text { Better } \\
\text { But not significantly }\end{array}$ \\
\hline Neural & $\begin{array}{l}\text { cs7 gain } \\
\text { cs } 10 \text { loss }\end{array}$ & $\uparrow N E F L$ GABRAI SYTI SLCI $2 A 5$ & & Poor \\
\hline
\end{tabular}

Abbreviations: ASCLI, achaete-scute complex homolog I; CD44, cluster of differentiation 44; CDKN2A, cyclin-dependent kinase inhibitor 2A; CHI3LI, chitinase3-like protein I; cs, chromosome; DCX, doublecortin; DLL3, delta-like 3; EGFR, epidermal growth factor receptor; EGFRvIII, mutated EGFR variant III; GABRAI, gamma-aminobutyric acid (GABA) receptor alpha I; GASI, growth arrest-specific I; GLII, glioma-associated oncogene I; HIF, hypoxia-inducible factor; IDHI, isocitrate dehydrogenase I (NADP+), soluble; JAG I, jagged I; LFNG, lunatic fringe (O-fucosylpeptide 3-beta- $\mathrm{N}$-acetylglucosaminlytransferase); MDM2, mouse double minute 2 homolog; MET, mesenchymal-epithelial transition factor; MERTK, MER proto-oncogene tyrosine kinase; NEFL, neurofilament, light polypeptide; NES, nestin; NFI, neurofibromatosis I; NFKB, nuclear factor kappa-light-chain-enhancer of activated B cells; NKX2-2, NK2 homeobox 2; NOTCH3, 3rd human notch homolog; OLIG2, oligodendrocyte lineage transcription factor 2; PDGFRA, platelet-derived growth factor receptor alpha; PI3K, phosphoinositide-3-kinase; PIK3CA, phosphatidylinositol-4,5-bisphosphate 3-kinase, catalytic subunit alpha; PIK3RI, PI3K regulatory subunit I; PTEN, phosphatase and tensin homolog; SLCI2A5, solute carrier family I2 (potassium/chloride transporter), member 5; SMO, smoothened; gas I, growth arrest specific I; SOX, Sry-related HMG box; SYTI, synaptotagmin I; TCF3/4, transcription factor 3 or 4; TCGA, The Cancer Genome Atlas; TNF, tumor necrosis factor; TMZ, temozolomide; PI3KCA, phosphatidylinositol-4,5-bisphosphate 3 kinase, catalytic subunit alpha; XRT, external beam radiation therapy; YKL40, chitinase-3-like protein I; VEGFR, vascular endothelial growth factor receptor.

(usually, headache, seizures, intracranial pressure, vision/ hearing issues, and even personality changes), making the window for early disease screening almost nonexistent. Imaging techniques, such as magnetic resonance imaging $(\mathrm{MRI})^{26} /$ magnetic resonance spectroscopy (MRS) $)^{27}$ are standard brain tumor diagnostics; these are clearly valuable but are limited to use after a tumor is suspected due to the aforementioned symptoms. Also, these techniques are extremely expensive, as is the entire brain tumor affliction. ${ }^{28}$ Such imaging modalities generally offer little histologic or prognostic information without employing specialized technologies such as various forms of positron emission tomagraphy, vessel architectural imaging, or stimulated Raman scattering. Beyond that, the only acceptable diagnoses are made, intra- or postoperatively, by pathology, which offers prognostic evaluations using immunohistochemistry or genetic techniques. ${ }^{29,30}$ Obviously, those tests require tumor access from surgery or biopsy, both intracranially invasive procedures. This is especially true for recurrent tumors (which occur with nearly all patients with high-grade gliomas), where imaging distinctions for remnant tumor, regrowth, or postradiation necrosis are difficult, ${ }^{31}$ and "pseudoprogression" (radiographic indications - usually, gadolinium enhancement - that appear as progressively growing tumor following radiation but later resolve or devolve into radiation necrosis) is another common problem. ${ }^{32,33}$ Tumor diagnostic/biomarker capabilities from an accessible compartment (blood/sera, urine, or saliva) would be a major advance for neuro-oncology, especially since there seem to be no circulating exfoliated brain tumor cells $^{34}$ (cerebral spinal fluid offers mixed results ${ }^{35,36}$ ). Thus, most biomarker efforts for GBM are geared towards the determination of recurrent disease, either in the context of standard of care treatment or in a clinical trial setting, with an emphasis on clinical-level assessments.

\section{Circulating biomarkers in glioblastoma - current state Proteins}

Most of the proteins previously identified as putative GBM biomarkers in blood may be more closely associated with the GBM and its treatment as markers of neurotrauma rather than being unequivocally related to the tumor itself. ${ }^{34}$ These proteins include glial fibrillary acidic protein (GFAP), vascular endothelial growth factor (VEGF), epidermal growth factor receptor (EGFR), basic fibroblast growth factor (bFGF), chitinase-3-like protein (CHI3L1) or YKL-40, and matrix metalloproteinase 9 (MMP9). Other factors that are known to be secreted by tumors or by immune cells following encounters with GBMs include transforming growth factor beta (TGF- $\beta$ ) family members ${ }^{37}$ as well as interleukin 10 (IL-10). ${ }^{38}$ So far, there has been no clinical validation of these serum-borne molecules as glioma biomarkers, but such research is ongoing. ${ }^{21}$

\section{Lipids}

There is little mention of circulating lipid species as putative biomarkers for GBMs, but 24S-hydroxycholesterol is among 
the lipids described as brain-specific that have been identified in the systemic blood circulation. ${ }^{39}$ However, hydroxycholesterol seems only to appear at high levels in serum following severe central nervous system (CNS) trauma and not necessarily in response to brain tumors. ${ }^{40}$

\section{Nucleic acids}

The levels of free nucleic acids in circulation is low and are difficult to detect due to the lack of protection from nucleases that are present in the circulating system. However, some potential biomarker candidates have been proposed. The circulating nucleic acid content in the serum or plasma of GBM patients consists of both deoxyribonucleic acid (DNA) and ribonucleic acid (RNA) species and was summarized in a review by Holdhoff et al. ${ }^{34}$ Briefly, the potential circulating tumor DNA biomarkers include methylated promoter regions of O-6-methyl-guanine-DNA methyltransferase (MGMT), cyclin-dependent kinase inhibitor $2 A$ (p16), death-associated protein kinase 1 (DAPK), and Ras association (RalGDS/ AF-6) domain family member 1 (RASSF1A). Others have also shown methylation of promoter regions in $M G M T$ and phosphatase and tensin homolog (PTEN); however, detection rates of these epigenetic changes were low in serum samples, or not detectable in the case of PTEN, compared with the astrocytic tumor samples. ${ }^{41}$ Additionally, the authors analyzed the loss of heterozygosity ( $\mathrm{LOH}$ ) of chromosomes $10 \mathrm{q}, 19 \mathrm{q}$, and $1 \mathrm{p}$ in oligodendroglial tumors and reported a higher percentage of $\mathrm{LOH}$ in the tumor tissue compared with the serum samples.

Different RNA species have also been detected in the circulation. EGFRvIII message was detected in platelets isolated from glioma patients. ${ }^{42}$ Circulating tumor microRNA (miRNA) has also been detected in the blood of GBM patients. ${ }^{34,43}$ In these studies, miRNA 21 (miR-21) and miRNA 128 (miR-128) were shown to be upregulated in the blood of GBM patients. The cohorts in these studies were relatively small, less than 20 patients at most, and an analysis for these molecules should be performed on a higher number of patients to provide more evidence of the use of these molecules as biomarkers. Additionally, it is unclear how small miRNAs are present in serum without some form of proteinaceous or membrane-enclosed protection. EVs may provide that protection.

\section{Extracellular vesicles in neuro-oncology: biology and potential biomarkers}

The term "exosome" was used to describe exfoliated microvesicles from the rat C-6 glioma line over 30 years ago $;{ }^{44}$ the vesicle sizes $(500-1,000 \mathrm{~nm})$ are what we would describe today as microvesicles, and their origin appears to be from the cell surface rather than endosomal. In those and other earlier studies ${ }^{45,46}$ it is unclear whether any controls were used for the fetal calf serum supplementing the cell culture medium as it is now known that the fetal bovine sera frequently used in cell culture contain extracellular vesicles. ${ }^{47}$ Those vesicles may be cleared by centrifugation (ie, $>100,000 \times g$ ) prior to inclusion of the sera in culture medium. We may have been the first to describe exosomes in brain tumor cell lines from both human and murine sources, ${ }^{48}$ and our studies were soon followed by important publications from Al-Nedawi et $\mathrm{al}^{49}$ and Skog et al. ${ }^{50}$ The former demonstrated the presence of glioma-specific mutant EGFR variant III (EGFRvIII) in EVs from both cell lines transfected with the mutant construct and from EVs in the sera of mice growing those EGFRvIII-expressing tumors. Incubation of glioma cells that did not express the mutant receptor with EVs from cells that did express it showed the transfer of EGFRvIII to the nonexpressing cells, with the concomitant activation of tumor-promoting signaling pathways associated with EGFRvIII signaling (extracellular signal-regulated kinase [ERK]/protein kinase B [AKT] phosphorylation, VEGF release). The latter paper demonstrated the presence of mRNAs and miRNAs in the sera of patients with GBMs that may be of diagnostic utility. A major advantage of nucleic acid technologies in regard to EVs is that they have standard preparation/handling conditions, and one has the ability to amplify signals from small amounts of starting material, which is not possible with other biologics (eg, proteins and lipids). The paper also showed that there was a differential distribution of specific messenger (m)RNAs in EVs compared with the relative amounts in the cells of origin and that a model mRNA could be passaged from cell to cell by EVs, resulting in translation of the mRNA to protein in the recipient cells. Among the mRNAs found in at least some patient serum EVs, was that encoding EGFRvIII as a tumor-specific marker. Finally, the EVs had angiogenic-promoting activities in human brain microvascular endothelial cells.

Our group later showed ${ }^{51}$ that EGFRvIII appeared on the surfaces of exosomes from EGFRvIII-expressing cells (along with heat shock proteins [HSPs] 70 and 27) and that EGFRvIII protein could be identified on EVs from the sera of patients with GBM; however, EGFR seemed to be present in sera EVs from both patients and healthy donors. Exosomes from a murine brain tumor line served as potent anticancer vaccines in a prophylactic setting but were largely 
ineffectual in a stringent therapeutic setting, suggesting that immune responses to tumor exosomes may be strongly context-dependent. We also performed the first proteomic survey of brain tumor exosomes in that study, identifying 36 distinct proteins, including endogenous murine retroviral Gag polyproteins. Those proteins are indicative of a retrovirus known to be involved in the progression of murine tumors by subversion of immune surveillance. ${ }^{52}$ This paper noted the immune dichotomy of brain tumor exosomes, demonstrating the presence of putative tumor antigens as well as a number of immunosuppressive entities.

Other articles of interest have shown that various brain tumor cell EVs (from GBMs, oligodendroglioma cells, and medulloblastoma cells) have activities and impacts on recipient cells that range from transformation of those cells ${ }^{53}$ to induction of apoptosis, ${ }^{54}$ along with growth promotion and migratory-driving activities. ${ }^{55}$ That paper $^{55}$ again showed the immune dichotomy of medulloblastoma exosomes in both immunosuppressive and immunostimulatory functions and identified almost 150 proteins in proteomic analyses. Furthermore, in other studies, ${ }^{56,57}$ exosomes/EVs isloated from hypoxic GBM cells enhanced GBM bioactivity and induced epithelial cells to secrete tumor-promotion factors, resulting in increased tumor growth, migration, and vascularization and angiogenesis. This involved paracrine signaling via tissue factor associated with EVs. Thus, exosomes/EVs can serve as transfer mechanisms for tumor survival and progression. ${ }^{56,57}$ Curiously, coagulopathies are part of the comorbidities associated with brain tumors, and tissue factor likely plays a significant part. While its presence on EVs is well known, the role of tissue factor in the context of EVs remains unclear ${ }^{56,58}$ and may represent an area into which therapeutic intervention can expand. ${ }^{59}$

There have been some extremely interesting publications describing the role of EVs in the biology of GBM, but the attempts to find clear and utilizable biomarkers are at early stages, and the field faces the same (lack of) outcomes that have plagued the cancer biomarker field for years - ie, large numbers of putative biomarkers on input, few approved entities on output. ${ }^{60,61}$ The development of useful, robust, and clinically viable biomarkers follows a complex, multiphase pathway with harsh go/no-go decisions and is fraught with difficulties that involve biology, bioinformatics, and current plus developing (and currently unforeseen) biotechnologies. ${ }^{62}$ However, as biomarkers are tied to clinical drug development platforms in the form of companion diagnostics, and as multiplexed systems may be necessary to validate drug efficacy, ${ }^{63} \mathrm{EVs}$ will likely play vital roles as reservoirs of multiple types of biologic materials suitable for biomarker assays.

As alluded to previously, EVs contain proteins, nucleic acids, lipids, and other metabolites that reflect their cells of origin; individual or multiple entities amongst those biomaterials may prove to be the identifiers of cancer cells in the host. As EVs are present in (and purifiable from) essentially all routinely obtainable biofluids, a distinct advantage in this scenario is that EVs bundle multiple potential biomarkers in one package. Thus, multiplexed assays that evaluate more than one biomaterial (eg, RNA and protein) could theoretically be performed on the same sample. The concept of "liquid biopsy" is often applied to circulating tumor cells $\mathrm{s}^{64}$ and more recently to circulating cell-free (cf)-DNA, ${ }^{65}$ but the numbers of circulating tumor cells are often low (or even nonexistent, in the cases of patients with GBM), and cf-DNA is largely nontumor in origin. EVs overcome these barriers in liquid biopsy - EVs are known to occur in elevated quantities in the blood of cancer patients, ${ }^{66-68}$ and the protein and nucleic acid contents can be tumor-specific. ${ }^{50,51,55,69,70}$ With this background, we will explore what is known and what may be possible concerning the use of EVs as biomarkers for GBM.

\section{Glioblastoma extracellular vesicle protein content}

Perhaps the best known and most specific protein marker for GBMs is EGFRvIII; this has been identified on both cell line and patient serum EVs. ${ }^{49-51}$ It is a tumor-specific mutation (also found in several other cancer types that overexpress EGFR) $)^{71}$ that deletes 267 amino acids from the extracellular domain, resulting in a truncated receptor that has no clear ligands but nonetheless signals constitutively. ${ }^{72,73}$ There are some discrepancies concerning the frequency of the mutant protein in GBM patient samples, perhaps resulting from differences in techniques and reagents, ${ }^{71}$ but estimates range from $25 \%$ to over $60 \%$. Thus, EGFRvIII seems to be an excellent putative biomarker from a diagnostic perspective, and its loss of expression may be a predictive biomarker if the protein is directly targeted. ${ }^{74}$ However, it is likely that evaluation of the protein status would be the most direct assessment, but access to antibody reagents is limited, resulting in the use of nucleic acid techniques for detection. ${ }^{75}$ There remains concern over the lack of correlation between polymerase chain reaction (PCR)-based detection of EGFR $I I I$ and the actual protein expression levels, ${ }^{71}$ so specificity and sensitivity issues may depend on the techniques employed. 
Gene amplification of EGFR is common in GBMs, as is overexpression of the protein (and with EGFRvIII expression in a subset of the EGFR-amplified tumors). ${ }^{76} \mathrm{We}^{51}$ and others ${ }^{49,77}$ have identified EGFR in EVs from GBM cells and patient sera, but it also appears to be in EVs from healthy donors ${ }^{51}$ and from nontumor cell lines, ${ }^{78,79}$ which begs the question of specificity. However, it may be a valuable biomarker tool as part of a multiparameter screening assay. ${ }^{77}$ In a very limited study, we also identified EGFR2/v-erb-b2 avian erythroblastic leukemia viral oncogene homolog 2 (ErbB2)/human epidermal growth factor receptor 2 (HER2) as a relatively specific marker for EVs, from patients with medulloblastoma. ${ }^{55}$ Perhaps 17\% (or more) of GBMs probed express HER2, ${ }^{80,81}$ so this protein may also be a useful biomarker, but it is unclear whether it is incorporated into EVs from healthy donors; much larger sample sizes are necessary to establish that correlation.

Of the other proteins listed above as putative circulating protein biomarkers for GBM, all but CHI3L/YKL-40 have been found in EVs (and the mRNA for YKL-40 was identified).$^{50}$ We had identified GFAP in medulloblastoma, but it has also been identified in healthy donor human plasma $\mathrm{EVs},{ }^{82}$ suggesting that it may not be tumor-specific. VEGF-A protein was identified in human GBM cell line EVs by antibody array, ${ }^{50}$ and so far, there appear to be no other normal cell EVs carrying it, but it is obviously not a tumor-specific marker. Li et al have identified 112 proteins in a proteomic analysis of $\mathrm{U} 251 \mathrm{MG}$ cell line-derived $\mathrm{EVs} ;{ }^{83}$ of those, all but one (myoferlin) had been previously identified in other EVs. Our group found bFGF (also called FGF2) in medulloblastoma $\mathrm{EVs},{ }^{55}$ suggesting that it may be a tumor marker, but again, it is not tumor-specific, as is true of MMP9, TGF- $\beta$, and IL-10. MMP9 mRNA was found in GBM EVs,${ }^{50}$ and the protein is present and active in ovarian cancer EVs. ${ }^{84}$ MMPs and other extracellular proteases play important roles in modifying the tumor microenvironment for angiogenesis, migration, and invasion, implicating EVs as major players in this important function.

GBMs leave their hosts profoundly immunosuppressed, ${ }^{85,86}$ and those effects are often linked to TGF- $\beta$ and IL-10. ${ }^{38}$ Both of these cytokines are produced by immune system cells but have been identified as EV components from both cancer and normal cell types. ${ }^{51,82}$ There may be a question regarding the normal background amounts of those cytokines in EVs as IL-10 was identified coming from mesenchymal stem cell EVs, ${ }^{82}$ and active TGF- $\beta$ from EVs of thymus cell origin may drive regulatory $\mathrm{T}$ cell phenotypes. ${ }^{87}$ Curiously, we saw what appeared to be latent forms of TGF- $\beta$ on serum EVs from patients with GBMs. ${ }^{51}$ Since active TGF- $\beta 1$ has a serum half-life of $<2$ minutes, ${ }^{88}$ transport of it in blood may rely on EVs.

Other tumor-specific mutations identified in brain tumors are in the isocitrate dehydrogenases 1 (IDH1) and 2 (IDH2). These mutations are far more prevalent in lower-grade gliomas (and secondary gliomas) than in the high grades (such as GBMs). ${ }^{89}$ While we identified IDH1 in the proteome of medulloblastoma $\mathrm{EVs},{ }^{55}$ the peptide sequence coverage did not include the potentially mutated regions. However, using an antibody specific for the IDH1 R132H mutant protein, Shao et $\mathrm{al}^{77}$ included the enzyme as part of a fourprotein GBM molecular signature to interrogate GBM cell line and patient sera EVs. This was part of a micro nuclear magnetic resonance (micro-NMR) device study that indicated such a molecular signature, coupled with an extremely sensitive instrument, could accurately type responders vs nonresponders in both animal models and patients receiving therapy for GBMs. This, again, strongly suggests that effective biomarkers may come in packages (such as EVs), utilizable as a panel, rather than as individual entities. ${ }^{90,91}$ It also suggests that despite the nanoscale of EVs, microfabrication of devices capable of interrogating such vesicles for clinical applications is truly possible.

\section{Glioblastoma extracellular vesicle lipid content}

While relatively little is known about the lipid components of EVs, ${ }^{92}$ we are learning more all the time. ${ }^{93}$ Nonetheless, almost nothing has been reported on the lipid contents of GBM EVs. 24S-hydroxycholesterol in serum has been associated with CNS trauma ${ }^{94}$ but not so with brain tumors; ${ }^{40}$ still, it is unclear whether the methods for extraction and identification of 24S-hydroxycholesterol would have encompassed a significant population of extracellular vesicles, ${ }^{95}$ and cholesterol is one of the highly enriched lipids in extracellular vesicles. ${ }^{93}$ Thus, there may be CNS-enriched (or specific) lipid markers on the EVs from CNS tumors, but their discovery is pending.

\section{Glioblastoma extracellular vesicle nucleic acid content}

The nucleic acid content of EVs is quite diverse. Skog et a ${ }^{50}$ were the first to report on the mRNA and miRNA content of GBM EVs. Balaj et al found that EVs from different types of CNS tumors contained single-stranded DNA, and both complementary (c)DNA and genomic DNA as well as retrotransposable elements. ${ }^{69} \mathrm{Li}$ et al reported that an abundance of miRNAs and small (presumably noncoding) 
RNAs are packaged in glioma EVs. ${ }^{83}$ miRNAs may be very useful sources of biomarker potential in GBMs, given the differences in the miRNA signature for healthy subjects and GBM patients. ${ }^{83,96,97}$

Although the nucleic acid content of EVs is diverse, there are only a few potential biomarkers that have been suggested to date. As with the protein content of GBMderived EVs, the EGFRvIII mRNA is also one of the most specific and well-known markers that has been detected in EVs. In their work, Skog et a ${ }^{50}$ purified EVs from the serum of GBM patients and detected EGFRvIII mRNA in 28\% of samples tested using PCR-based methods. The percentage of EGFRvIII mRNA compared with total RNA in EVs is consistent with the percentage of GBM patients who are positive for this particular mutation, ie, about $30 \%$ of GBM patients have the EGFRvIII mutation. The detection of EGFRvIII RNA in glioma EVs was also reported by Nilsson et al, and they found that EVs are used to transfer the message to recipient cells. ${ }^{42}$ The work of Chen et al is promising in its potential to use the detection of the IDH1 mutation in CSF as a biomarker, especially considering the sensitivity of this technique. $^{70}$ The authors used very sensitive PCR methods to detect and quantify wild-type and mutant IDH1 transcripts in EVs isolated from the CSF of glioma patients and found higher levels of mutant IDH1 in the CSF of patients with gliomas compared with healthy controls. The sensitivity of the technique is evident considering that IDH1 mutants are relatively rare in patients with GBM. ${ }^{98}$ However, the source of material (CSF) is not a prime candidate for serial evaluation of biomarker status.

As mentioned above, Li et $\mathrm{al}^{83}$ detected several different types of RNA in glioma EVs. Of particular interest, is their finding that in the U251 cell line, derived EV miR-21 was detected in high levels, consistent with Skog et al. ${ }^{50}$ This finding complements the results from Skog et al, also showing a high expression of miR-21 in serum EVs from patients with GBM. ${ }^{40}$ The results of both of these groups are consistent with reports that miR-21 is highly overexpressed in glioma tissue and suggest that miR-21 has clear potential as a biomarker in GBMs, ${ }^{97,99}$ particularly in the context of other putative markers. It should be pointed out that miR-21 is not specific for GBMs and is also found in the EVs of healthy donors, ${ }^{100}$ so "context and community" will likely be important to determine the utility of miRNAs as biomarkers. As sophisticated, sensitive PCR methodologies become robust and more widely usable in a diagnostic setting, it is conceivable that the detection of other highly abundant GBM-specific miRNAs in biofluid
EVs will be possible and may provide additional biomarker candidates.

\section{Other aspects of extracellular vesicles relatable to biomarkers EV surfaces as interrogable landscapes}

The surfaces of EVs contain a great deal of information relative to the sizes of these tiny vesicles and considering the relatively limited internal volumes of them. Various aspects of EV surfaces may reveal much about their abilities to differentially represent the presence of tumor vs normal cells, or the changes in tumor cells due to treatment.

\section{Glycome}

The surfaces of cancer cells are often differentially glycosylated compared with their normal cell counterparts, ${ }^{101}$ thus suggesting that tumor glycomes may distinguish tumor cells from normal cells. Technologies for carbohydrate analyses are progressing, including better separation and mass spectrometry identification, as well as microarraystyle high-throughput methods. ${ }^{102}$ The glycosylated surfaces of EVs, either in the form of glycoproteins, glycolipids, or surface-bound carbohydrates, are ripe for this type of probing.

Recently, Batista et al utilized a lectin array to study the glycomes of EVs harvested from several different sources. ${ }^{103}$ They showed differences between the cell surface glycomes and the resulting EV glycomes, which tended to be conserved, although there were distinguishing features in the gross glycomic compositions. Urinary exosome surface glycosylation has also been proven to distinguish between EVs from healthy donors vs those with polycystic kidney disease, ${ }^{104}$ suggesting that these surface phenomena may harbor carbohydrate biomarkers. We recently reported that particular antibodies that recognize the brain tumor-specific gangliosides 3'-isoLM1 and/or $3^{\prime}, 6^{\prime}$-isoLD $1^{105}$ would bind to glioma exosomes, ${ }^{106}$ demonstrating that such antigens could be present on circulating EVs as biomarkers. This is a relatively unexplored area in EV biology, but it may hold great promise as a means for identifying pathology-related circulating EVs.

\section{Proteome}

The EV surface proteome is likely to contain proteins (in particular, glycoproteins) that reflect the cancerous state. GBM "stem cells" were used to profile cell surface glycoproteins whose expression was upregulated on the "neurospheres" stem cells compared with classic, long-term adherent cultures. ${ }^{107}$ Receptor-type tyrosine protein phosphatase $\zeta$ (phosphacan), 
tenascin-C, chondroitin sulfate proteoglycan NG2 (CSPG4), podocalyxin-like protein 1 , and cluster of differentiation (CD)90 were upregulated (and CD44 was comparatively downregulated). Of those glycoproteins, tenascin C, CSPG4, and CD44 have been identified in EVs, ${ }^{82}$ and there is an antibody (81C6) that specifically recognizes brain tumor tenascin $\mathrm{C}^{108}$ as well as a potential therapeutic antibody against CSPG4. ${ }^{109}$ Thus, the stem cell portions of GBMs may have surface markers that could be released in EVs; due to the association of stem cells with tumor therapeutic resistance, these may be valuable markers, as harbingers of impending recurrence.

As mentioned above, our group identified EGFRvIII on glioma cell EV surfaces, along with HSPs 27 and 70, ${ }^{51}$ and later, we showed HSP90 on medulloblastoma EV surfaces. ${ }^{55}$ The presence of HSPs on brain tumor EV surfaces fits with the demonstration of the HSPs and other chaperones on the surfaces of a variety of brain tumor cells. ${ }^{48,110}$ As these proteins are typically regarded as intracellularly localized, these data suggest that location may relate to biomarker status, with tumors altering the "standard" localizations of proteins. Newer technologies, such as nanoparticle tracking analysis (NTA) with fluorescent (usually antibody) staining, allows for the specific quantification of labeled vesicles within the overall population of vesicles present, thus allowing for both the detection and enumeration of putative tumor or pathologic EVs in a mixed field of vesicles (eg, such as from serum ${ }^{111}$ or from urine ${ }^{112}$ ). Another use of antibody detection for both surface and internal EV content was mentioned previously, where using a microfluidic NMR chip, GBM EVs were labeled (or lysed and internal materials labeled) with targeted magnetic nanoparticles against a four-protein signature (EGFR, EGFRvIII, podoplanin [PDPN], and IDH1 mutant). ${ }^{77}$ This rendered the EVs superparamagnetic and yielded faster proton decay rates for enhanced NMR signal. While none of the markers individually had high sensitivity (but overall good specificity), the combination of the four markers resulted in both high sensitivity and specificity, and the ability to quantify EVs at several logs better than current technologies. A major message here is that multiple biomarkers may be able to overcome the limitations of one or a few.

\section{Surface antigens}

One interesting host response to tumor EVs is the antibody response against presumed surface antigens. This has been shown with model or known antigens "loaded" into EVs, ${ }^{113,114}$ but we have also shown high-titer murine responses against naturally occurring brain tumor antigens, ${ }^{51,106}$ these responses occur very quickly and in the absence of adjuvant.
Conversely, tumor antigens (eg, HER2) on the surfaces of circulating tumor-derived EVs have the capacity to bind and effectively titrate out therapeutic antibodies, such as trastuzumab, potentially reducing the effectiveness of such drugs. ${ }^{115}$ There appear to be natural antibodies that recognize reticulocyte EVs, with potential involvement in the elimination of apoptotic bodies. ${ }^{116}$ We have shown that circulating EVs from the sera of patients with GBMs carry substantial amounts of bound antibody; those antibodies may be "eluted" from the EVs, and at least some fraction of the antibodies recognize tumor antigens from GBM cell lines, in Western blots. ${ }^{106}$ This raises the possibility that antibodies on circulating tumor EVs may be able to reveal the presence of tumor antigens, which may produce a signature on high-density protein or peptide arrays alerting of tumor presence. From a predictive perspective, one issue would be our lack of knowledge of the clearance or half-lives of such antibodies, so there may be more value in them from an initial diagnostic perspective.

\section{Glioblastoma extracellular vesicle biologic activities with biomarker potential}

One should consider the concept that particular molecular entities of EVs may be difficult to identify and ascertain as recognizably different between tumors and normal cell types. However, the collection of materials may have endemic, measurable activities that could distinguish between pathologic and normal states; in a further connection of tumorigenicity to a measurable readout, EVs may be able induce responses in model cell types that could reveal the presence of tumor and perhaps even, in some situations, an "aggressiveness" measure of the tumor. The following are some possible areas of assay development.

\section{Metabolic}

Biologic/metabolic enzymes represent about $25 \%$ of the proteins found in brain tumor exosomes ${ }^{51,55}$ and glycolytic enzymes are among the top ten proteins identified in EVs. ${ }^{117}$ Ronquist et al recently demonstrated that prostasomes (EVs released from the prostate gland into seminal fluid) can functionally generate adenosine triphosphate (ATP) from substrates such as glucose or fructose, ${ }^{118}$ indicating that EVs have intrinsic metabolic capacity. We recently showed that EVs from the sera of patients with GBM possessed the tumorassociated enzyme pyruvate kinase M2 (PKM2) necessary for the Warburg effect and that the addition of EVs from GBM cells increased the expression of various metabolic enzymes in those recipient cells. ${ }^{106}$ One potential effect of the increased enzyme quantities could be increased measurable 
metabolic output; for instance, the addition of GBM EVs to GBM (or other reporter) cells might drive lactate release, suggesting that such assays may reveal the presence of tumor EVs by surrogate cellular-based assays.

\section{Attractant}

We previously demonstrated that medulloblastoma cells migrated towards EVs used as attractants in Boyden chamber/ transwell migration assays, ${ }^{55}$ and this has proven true for EVs of other tumor types. ${ }^{119}$ While this likely has more biologic than diagnostic significance, certain elements of EV activity (eg, extracellular proteolytic activity of MMP9) may have useful, measurable outputs in the appropriate assays.

\section{Signaling}

EVs are known to transport phosphoproteins with putative signaling potential, ${ }^{120,121}$ and GBM EVs can alter signaling pathways within recipient cells. ${ }^{122}$ In similar assays, our unpublished data show that EVs from "stressed" GBM cells (ie, those induced to undergo the unfolded protein response) $)^{123}$ drive more extensive changes in receptor tyrosine kinase signaling pathways on recipient GBM cells than do those EVs from "unstressed" cells (not shown). One could imagine a standard cell line assay, where EVs from patient body fluids were incubated with the model cell line, which was then lysed and assayed for specific (ie, limited) changes in phosphoprotein status indicative of tumor EV-driven phenotypic alterations. It is conceivable that EVs themselves could be lysed and exposed to phosphoantibody arrays that might reveal the presence of high levels of tumor-associated phosphoproteins. Other EV protein posttranslational modifications that may indicate tumor presence may include glycosylation (see above), ubiquitination, ${ }^{124}$ citrullination, ${ }^{125}$ acylation, ${ }^{126}$ and other lipid modifications. We are likely just scratching the surface in understanding posttranslational modifications and their impacts on cargo loading into EVs, and potential downstream biologic significance.

\section{Discussion}

EVs show great promise as reservoirs of biomarkers for patients with glioblastoma. The presence of EVs in every biofluid allows for theoretically easy access in minimally or noninvasive fashion, and the assortment of biomolecules surely provides a signature for the presence of disease or of change in the disease state. In few cases are the components of that signature truly tumor-specific (in the cases of GBMs, perhaps EGFRvIII and IDH mutants are tumor-restricted). Thus, we may need to quantify potential (combinations of) biomarkers, and possibly their locations in or on EVs, to distinguish the signature as distinct from normal tissue EVs. That signature may be only readable through a translation via bioassays rather than by the direct identification of "letters" or "words" in the signature. There is a great need for biomarker development in neuro-oncology since our current imaging systems often fail to provide adequate information concerning recurrent tumor growth or current biological status of either the primary or recurrent tumor. This paucity of information tends to drive a fairly uniform treatment regimen for every patient and thus, fails to account for a personalized approach to monitoring disease and individual responses to therapy. It may be that these circulating "fat balls" can provide that window into the presence and status of the tumor that will give us a therapeutic edge in the treatment of these devastating diseases.

\section{Acknowledgments}

The authors wish to thank Dr Bette K Kleinschmidt-DeMasters, from the Department of Pathology at the University OfColorado School Of Medicine, for her histological contributions to this paper. This work was supported by an award from the National Institutes of Health 1R01EB016378-01A1 and the Cancer League of Colorado (to MWG).

\section{Disclosure}

The authors report no conflicts of interest in this work.

\section{References}

1. Dolecek TA, Propp JM, Stroup NE, Kruchko C. CBTRUS statistical report: primary brain and central nervous system tumors diagnosed in the United States in 2005-2009. Neuro Oncol. 2012;14 Suppl 5:v1-v49.

2. Grossman SA, Ye X, Piantadosi S, et al; NABTT CNS Consortium. Survival of patients with newly diagnosed glioblastoma treated with radiation and temozolomide in research studies in the United States. Clin Cancer Res. 2010;16(8):2443-2449.

3. Bradley S, Sherwood PR, Donovan HS, et al. I could lose everything: understanding the cost of a brain tumor. $J$ Neurooncol. 2007;85(3): 329-338.

4. Pickard JD, Bailey S, Sanderson H, Rees M, Garfield JS. Steps towards cost-benefit analysis of regional neurosurgical care. BMJ. 1990;301(6753):629-635.

5. Heimberger AB, Sampson JH. The PEPvIII-KLH (CDX-110) vaccine in glioblastoma multiforme patients. Expert Opin Biol Ther. 2009;9(8): 1087-1098.

6. Das S, Marsden PA. Angiogenesis in glioblastoma. $N$ Engl J Med. 2013;369(16):1561-1563.

7. Dea N, Fournier-Gosselin MP, Mathieu D, Goffaux P, Fortin D. Does extent of resection impact survival in patients bearing glioblastoma? Can J Neurol Sci. 2012;39(5):632-637.

8. Lacroix M, Abi-Said D, Fourney DR, et al. A multivariate analysis of 416 patients with glioblastoma multiforme: prognosis, extent of resection, and survival. J Neurosurg. 2001;95(2):190-198.

9. Cloughesy TF, Cavenee WK, Mischel PS. Glioblastoma: from molecular pathology to targeted treatment. Annu Rev Pathol. Epub August 5, 2013. 
10. Denysenko T, Gennero L, Juenemann C, et al. Heterogeneous phenotype of human glioblastoma. In vitro study. Cell Biochem Funct. Epub July 8, 2013.

11. Thomas RP, Xu LW, Lober RM, Li G, Nagpal S. The incidence and significance of multiple lesions in glioblastoma. $J$ Neurooncol. 2013;112(1):91-97.

12. Arakawa Y, Mizowaki T, Murata D, et al. Retrospective analysis of bevacizumab in combination with Ifosfamide, Carboplatin, and Etoposide in patients with second recurrence of glioblastoma. Neurol Med Chir (Tokyo). 2013;53(11):779-785.

13. Tanaka S, Louis DN, Curry WT, Batchelor TT, Dietrich J. Diagnostic and therapeutic avenues for glioblastoma: no longer a dead end? Nat Rev Clin Oncol. 2013;10(1):14-26.

14. Graner MW. Brain tumor exosomes and microvesicles: pleiotropic effects from tiny cellular surrogates. In: Garami M, editor. Molecular Targets of CNS Tumors. Rijeka: InTech; 2012.

15. Raposo G, Stoorvogel W. Extracellular vesicles: exosomes, microvesicles, and friends. J Cell Biol. 2013;200(4):373-383.

16. Martins VR, Dias MS, Hainaut P. Tumor-cell-derived microvesicles as carriers of molecular information in cancer. Curr Opin Oncol. 2013;25(1):66-75.

17. Lässer C, Eldh M, Lötvall J. Isolation and characterization of RNAcontaining exosomes. J Vis Exp. 2012;(59):e3037.

18. Théry C, Amigorena S, Raposo G, Clayton A. Isolation and characterization of exosomes from cell culture supernatants and biological fluids. Curr Protoc Cell Biol. 2006; Chapter 3:Unit 3.22.

19. Properzi F, Logozzi M, Fais S. Exosomes: the future of biomarkers in medicine. Biomark Med. 2013;7(5):769-778.

20. Rak J. Extracellular vesicles - biomarkers and effectors of the cellular interactome in cancer. Front Pharmacol. 2013;4:21.

21. Farias-Eisner G, Bank AM, Hwang BY, et al. Glioblastoma biomarkers from bench to bedside: advances and challenges. $\mathrm{Br} J$ Neurosurg. 2012;26(2):189-194.

22. Biomarkers Definitions Working Group. Biomarkers and surrogate endpoints: preferred definitions and conceptual framework. Clin Pharmacol Ther. 2001;69(3):89-95.

23. Ohgaki H, Kleihues P. Genetic pathways to primary and secondary glioblastoma. Am J Pathol. 2007;170(5):1445-1453.

24. Johnson DR, Galanis E. Incorporation of prognostic and predictive factors into glioma clinical trials. Curr Oncol Rep. 2013;15(1) $56-63$.

25. Verhaak RG, Hoadley KA, Purdom E, et al; Cancer Genome Atlas Research Network. Integrated genomic analysis identifies clinically relevant subtypes of glioblastoma characterized by abnormalities in PDGFRA, IDH1, EGFR, and NF1. Cancer Cell. 2010;17(1) 98-110.

26. Price SJ. The role of advanced MR imaging in understanding brain tumour pathology. Br J Neurosurg. 2007;21(6):562-575.

27. Shah N, Sattar A, Benanti M, Hollander S, Cheuck L. Magnetic resonance spectroscopy as an imaging tool for cancer: a review of the literature. J Am Osteopath Assoc. 2006;106(1):23-27.

28. Bradley CJ, Yabroff KR, Dahman B, Feuer EJ, Mariotto A, Brown ML. Productivity costs of cancer mortality in the United States: 2000-2020. J Natl Cancer Inst. 2008;100(24):1763-1770.

29. Takei H, Bhattacharjee MB, Rivera A, Dancer Y, Powell SZ. New immunohistochemical markers in the evaluation of central nervous system tumors: a review of 7 selected adult and pediatric brain tumors. Arch Pathol Lab Med. 2007;131(2):234-241.

30. Nakamura M, Shimada K, Ishida E, Nakase H, Konishi N. Genetic analysis to complement histopathological diagnosis of brain tumors. Histol Histopathol. 2007;22(3):327-335.

31. Czernicki T, Szeszkowski W, Marchel A, Gołebiowski M. Spectral changes in postoperative MRS in high-grade gliomas and their effect on patient prognosis. Folia Neuropathol. 2009;47(1):43-49.

32. Van Mieghem E, Wozniak A, Geussens Y, et al. Defining pseudoprogression in glioblastoma multiforme. Eur J Neurol. 2013;20(10): 1335-1341.
33. Tran DK, Jensen RL. Treatment-related brain tumor imaging changes: So-called "pseudoprogression" vs tumor progression: Review and future research opportunities. Surg Neurol Int. 2013;4(Suppl 3): S129-S135.

34. Holdhoff M, Yovino SG, Boadu O, Grossman SA. Blood-based biomarkers for malignant gliomas. J Neurooncol. 2013;113(3):345-352.

35. Qian X, Goumnerova LC, De Girolami U, Cibas ES. Cerebrospinal fluid cytology in patients with ependymoma: a bi-institutional retrospective study. Cancer. 2008;114(5):307-314.

36. Kajikawa H, Ohta T, Ohshiro H, Harada K, Ishikawa S. Cerebrospinal fluid cytology in patients with brain tumors; a simple method using the cell culture technique. Acta Cytol. 1977;21(1):162-167.

37. Kaminska B, Kocyk M, Kijewska M. TGF beta signaling and its role in glioma pathogenesis. Adv Exp Med Biol. 2013;986:171-187.

38. Rolle CE, Sengupta S, Lesniak MS. Mechanisms of immune evasion by gliomas. Adv Exp Med Biol. 2012;746:53-76.

39. Björkhem I, Lütjohann D, Diczfalusy U, Ståhle L, Ahlborg G, Wahren J. Cholesterol homeostasis in human brain: turnover of 24S-hydroxycholesterol and evidence for a cerebral origin of most of this oxysterol in the circulation. J Lipid Res. 1998;39(8): 1594-1600.

40. Bretillon L, Sidén A, Wahlund LO, et al. Plasma levels of 24S-hydroxycholesterol in patients with neurological diseases. Neurosci Lett. 2000;293(2):87-90.

41. Lavon I, Refael M, Zelikovitch B, Shalom E, Siegal T. Serum DNA can define tumor-specific genetic and epigenetic markers in gliomas of various grades. Neuro Oncol. 2010;12(2):173-180.

42. Nilsson RJ, Balaj L, Hulleman E, et al. Blood platelets contain tumorderived RNA biomarkers. Blood. 2011;118(13):3680-3683.

43. Roth P, Wischhusen J, Happold C, et al. A specific miRNA signature in the peripheral blood of glioblastoma patients. $J$ Neurochem. 2011;118(3):449-457.

44. Trams EG, Lauter CJ, Salem N Jr, Heine U. Exfoliation of membrane ecto-enzymes in the form of micro-vesicles. Biochim Biophys Acta. 1981;645(1):63-70.

45. Bastida E, Ordinas A, Escolar G, Jamieson GA. Tissue factor in microvesicles shed from U87MG human glioblastoma cells induces coagulation, platelet aggregation, and thrombogenesis. Blood. 1984;64(1):177-184.

46. Kassis S, Lauter CJ, Stojanov M, Salem N. Exfoliation of the betaadrenergic receptor and the regulatory components of adenylate cyclase by cultured rat glioma C6 cells. Biochim Biophys Acta. 1986;886(3): 474-482.

47. Menon S, Isenberg DA. Fetal calf serum in growth medium obscures the detection of early anticardiolipin antibody secreting clones. J Immunol Methods. 1995;186(1):65-70.

48. Graner MW, Cumming RI, Bigner DD. The heat shock response and chaperones/heat shock proteins in brain tumors: surface expression, release, and possible immune consequences. J Neurosci. 2007;27(42): 11214-11227.

49. Al-Nedawi K, Meehan B, Micallef J, et al. Intercellular transfer of the oncogenic receptor EGFRvIII by microvesicles derived from tumour cells. Nat Cell Biol. 2008;10(5):619-624.

50. Skog J, Würdinger T, van Rijn S, et al. Glioblastoma microvesicles transport RNA and proteins that promote tumour growth and provide diagnostic biomarkers. Nat Cell Biol. 2008;10(12):1470-1476.

51. Graner MW,Alzate O, Dechkovskaia AM, et al. Proteomic and immunologic analyses of brain tumor exosomes. FASEB J. 2009;23(5):1541-1557.

52. Mangeney M, Pothlichet J, Renard M, Ducos B, Heidmann T Endogenous retrovirus expression is required for murine melanoma tumor growth in vivo. Cancer Res. 2005;65(7):2588-2591.

53. Antonyak MA, Li B, Boroughs LK, et al. Cancer cell-derived microvesicles induce transformation by transferring tissue transglutaminase and fibronectin to recipient cells. Proc Natl Acad Sci U SA. 2011;108(12): $4852-4857$.

54. Lo Cicero A, Schiera G, Proia P, et al. Oligodendroglioma cells shed microvesicles which contain TRAIL as well as molecular chaperones and induce cell death in astrocytes. Int J Oncol. 2011;39(6):1353-1357. 
55. Epple LM, Griffiths SG, Dechkovskaia AM, et al. Medulloblastoma exosome proteomics yield functional roles for extracellular vesicles. PLoS One. 2012;7(7):e42064.

56. Svensson KJ, Kucharzewska P, Christianson HC, et al. Hypoxia triggers a proangiogenic pathway involving cancer cell microvesicles and PAR-2-mediated heparin-binding EGF signaling in endothelial cells. Proc Natl Acad Sci U S A. 2011;108(32): 13147-13152.

57. Kucharzewska P, Christianson HC, Welch JE, et al. Exosomes reflect the hypoxic status of glioma cells and mediate hypoxia-dependent activation of vascular cells during tumor development. Proc Natl Acad Sci U S A. 2013;110(18):7312-7317.

58. Magnus N, D’Asti E, Garnier D, Meehan B, Rak J. Brain neoplasms and coagulation. Semin Thromb Hemost. 2013;39(8):881-895.

59. Mitroulis I, Kambas K, Anyfanti P, Doumas M, Ritis K. The multivalent activity of the tissue factor-thrombin pathway in thrombotic and nonthrombotic disorders as a target for therapeutic intervention. Expert Opin Ther Targets. 2011;15(1):75-89.

60. Srivastava S. Cancer biomarker discovery and development in gastrointestinal cancers: early detection research network-a collaborative approach. Gastrointest Cancer Res. 2007;1(4 Suppl 2):S60-S63.

61. Sawyers CL. The cancer biomarker problem. Nature. 2008;452(7187): 548-552.

62. Prensner JR, Chinnaiyan AM, Srivastava S. Systematic, evidence-based discovery of biomarkers at the NCI. Clin Exp Metastasis. 2012;29(7): 645-652.

63. Jørgensen JT. A changing landscape for companion diagnostics. Expert Rev Mol Diagn. 2013;13(7):667-669.

64. Alix-Panabières $\mathrm{C}$, Pantel K. Circulating tumor cells: liquid biopsy of cancer. Clin Chem. 2013;59(1):110-118.

65. Crowley E, Di Nicolantonio F, Loupakis F, Bardelli A. Liquid biopsy: monitoring cancer-genetics in the blood. Nat Rev Clin Oncol. 2013;10(8):472-484.

66. Taylor DD, Gercel-Taylor C. MicroRNA signatures of tumor-derived exosomes as diagnostic biomarkers of ovarian cancer. Gynecol Oncol. 2008;110(1):13-21.

67. Jansa R, Sustar V, Frank M, et al. Number of microvesicles in peripheral blood and ability of plasma to induce adhesion between phospholipid membranes in 19 patients with gastrointestinal diseases. Blood Cells Mol Dis. 2008;41(1):124-132.

68. Galindo-Hernandez O, Villegas-Comonfort S, Candanedo F, et al. Elevated concentration of microvesicles isolated from peripheral blood in breast cancer patients. Arch Med Res. 2013;44(3):208-214.

69. Balaj L, Lessard R, Dai L, et al. Tumour microvesicles contain retrotransposon elements and amplified oncogene sequences. Nat Commun. 2011;2:180.

70. Chen WW, Balaj L, Liau LM, et al. BEAMing and droplet digital PCR analysis of mutant IDH1 mRNA in glioma patient serum and cerebrospinal fluid extracellular vesicles. Mol Ther Nucleic Acids. 2013;2:e109.

71. Gan HK, Cvrljevic AN, Johns TG. The epidermal growth factor receptor variant III (EGFRvIII): where wild things are altered. FEBS J. 2013;280(21):5350-5370.

72. Batra SK, Castelino-Prabhu S, Wikstrand CJ, et al. Epidermal growth factor ligand-independent, unregulated, cell-transforming potential of a naturally occurring human mutant EGFRvIII gene. Cell Growth Differ. 1995;6(10):1251-1259.

73. Wikstrand CJ, Reist CJ, Archer GE, Zalutsky MR, Bigner DD. The class III variant of the epidermal growth factor receptor (EGFRvIII): characterization and utilization as an immunotherapeutic target. J Neurovirol. 1998;4(2):148-158.

74. Sampson JH, Heimberger AB, Archer GE, et al. Immunologic escape after prolonged progression-free survival with epidermal growth factor receptor variant III peptide vaccination in patients with newly diagnosed glioblastoma. J Clin Oncol. 2010;28(31):4722-4729.

75. Yoshimoto K, Dang J, Zhu S, et al. Development of a real-time RT-PCR assay for detecting EGFRvIII in glioblastoma samples. Clin Cancer Res. 2008;14(2):488-493.
76. Hegi ME, Rajakannu P, Weller M. Epidermal growth factor receptor: a re-emerging target in glioblastoma. Curr Opin Neurol. 2012;25(6): 774-779.

77. Shao H, Chung J, Balaj L, et al. Protein typing of circulating microvesicles allows real-time monitoring of glioblastoma therapy. Nat Med. 2012;18(12):1835-1840.

78. Sanderson MP, Keller S, Alonso A, Riedle S, Dempsey PJ, Altevogt P. Generation of novel, secreted epidermal growth factor receptor (EGFR/ ErbB1) isoforms via metalloprotease-dependent ectodomain shedding and exosome secretion. J Cell Biochem. 2008;103(6):1783-1797.

79. Kim HS, Choi DY, Yun SJ, et al. Proteomic analysis of microvesicles derived from human mesenchymal stem cells. J Proteome Res. 2012;11(2):839-849.

80. Forseen SE, Potti A, Koka V, Koch M, Fraiman G, Levitt R. Identification and relationship of HER-2/neu overexpression to shortterm mortality in primary malignant brain tumors. Anticancer Res. 2002;22(3):1599-1602.

81. Mineo JF, Bordron A, Quintin-Roué I, et al. Recombinant humanised anti-HER2/neu antibody (Herceptin) induces cellular death of glioblastomas. Br J Cancer. 2004;91(6):1195-1199.

82. microvesicles.org [homepage on the Internet]. Vesiclepedia. 2013. Available from: microvesicles.org. Accessed December 13, 2013.

83. Li CC, Eaton SA, Young PE, et al. Glioma microvesicles carry selectively packaged coding and non-coding RNAs which alter gene expression in recipient cells. RNA Biol. 2013;10(8):1333-1344.

84. Giusti I, D’Ascenzo S, Millimaggi D, et al. Cathepsin B mediates the $\mathrm{pH}$-dependent proinvasive activity of tumor-shed microvesicles. Neoplasia. 2008;10(5):481-488.

85. Fecci PE, Mitchell DA, Whitesides JF, et al. Increased regulatory T-cell fraction amidst a diminished CD4 compartment explains cellular immune defects in patients with malignant glioma. Cancer Res. 2006;66(6):3294-3302.

86. Waziri A. Glioblastoma-derived mechanisms of systemic immunosuppression. Neurosurg Clin N Am. 2010;21(1):31-42.

87. Wang GJ, Liu Y, Qin A, et al. Thymus exosomes-like particles induce regulatory T cells. J Immunol. 2008;181(8):5242-5248.

88. Philip A, O'Connor-McCourt MD. Interaction of transforming growth factor-beta 1 with alpha 2-macroglobulin. Role in transforming growth factor-beta 1 clearance. J Biol Chem. 1991;266(33):22290-22296.

89. Cohen AL, Holmen SL, Colman H. IDH1 and IDH2 mutations in gliomas. Curr Neurol Neurosci Rep. 2013;13(5):345.

90. Xiao Z, Prieto D, Conrads TP, Veenstra TD, Issaq HJ. Proteomic patterns: their potential for disease diagnosis. Mol Cell Endocrinol. 2005;230(1-2):95-106.

91. Yurkovetsky ZR, Linkov FY, E Malehorn D, Lokshin AE. Multiple biomarker panels for early detection of ovarian cancer. Future Oncol. 2006;2(6):733-741.

92. Pilzer D, Gasser O, Moskovich O, Schifferli JA, Fishelson Z. Emission of membrane vesicles: roles in complement resistance, immunity and cancer. Springer Semin Immunopathol. 2005;27(3):375-387.

93. Llorente A, Skotland T, Sylvänne T, et al. Molecular lipidomics of exosomes released by PC-3 prostate cancer cells. Biochim Biophys Acta. 2013;1831(7):1302-1309.

94. Weiner MF, Vega GL, Diaz-Arrastia R, et al. Plasma 24S-hydroxycholesterol and other oxysterols in acute closed head injury. Brain Inj. 2008;22(7-8):611-615.

95. Dzeletovic S, Breuer O, Lund E, Diczfalusy U. Determination of cholesterol oxidation products in human plasma by isotope dilution-mass spectrometry. Anal Biochem. 1995;225(1):73-80.

96. Mizoguchi M, Guan Y, Yoshimoto K, et al. MicroRNAs in human malignant gliomas. J Oncol. 2012;2012:732874.

97. Conti A, Aguennouz M, La Torre D, et al. miR-21 and 221 upregulation and miR-181b downregulation in human grade II-IV astrocytic tumors. J Neurooncol. 2009;93(3):325-332.

98. Gupta R, Webb-Myers R, Flanagan S, Buckland ME. Isocitrate dehydrogenase mutations in diffuse gliomas: clinical and aetiological implications. J Clin Pathol. 2011;64(10):835-844. 
99. Hermansen SK, Dahlrot RH, Nielsen BS, Hansen S, Kristensen BW. MiR-21 expression in the tumor cell compartment holds unfavorable prognostic value in gliomas. $J$ Neurooncol. 2013;111(1):71-81.

100. Tanaka Y, Kamohara H, Kinoshita K, et al. Clinical impact of serum exosomal microRNA-21 as a clinical biomarker in human esophageal squamous cell carcinoma. Cancer. 2013;119(6):1159-1167.

101. Abbott KL, Pierce JM. Lectin-based glycoproteomic techniques for the enrichment and identification of potential biomarkers. Methods Enzymol. 2010;480:461-476.

102. Rakus JF, Mahal LK. New technologies for glycomic analysis: toward a systematic understanding of the glycome. Annu Rev Anal Chem (Palo Alto Calif). 2011;4:367-392.

103. Batista BS, Eng WS, Pilobello KT, Hendricks-Muñoz KD, Mahal LK Identification of a conserved glycan signature for microvesicles. J Proteome Res. 2011;10(10):4624-4633.

104. Gerlach JQ, Krüger A, Gallogly S, et al. Surface glycosylation profiles of urine extracellular vesicles. PLoS One. 2013;8(9):e74801.

105. Wikstrand CJ, Fredman P, Svennerholm L, Bigner DD. Detection of glioma-associated gangliosides GM2, GD2, GD3, 3'-isoLM1 3',6'isoLD1 in central nervous system tumors in vitro and in vivo using epitope-defined monoclonal antibodies. Prog Brain Res. 1994;101: 213-223.

106. Graner MW, Epple LM, Dusto NL, et al. Circulating exosomes as new biomarkers for brain disease and injury: Proceedings of SPIE 8723 Sensing Technologies for Global Health, Military Medicine, and Environmental Monitoring III; May 29, 2013; Baltimore, MD.

107. He J, Liu Y, Xie X, et al. Identification of cell surface glycoprotein markers for glioblastoma-derived stem-like cells using a lectin microarray and LC-MS/MS approach. J Proteome Res. 2010;9(5): 2565-2572.

108. Kurpad SN, Zhao XG, Wikstrand CJ, Batra SK, McLendon RE, Bigner DD. Tumor antigens in astrocytic gliomas. Glia. 1995;15(3): 244-256.

109. Poli A, Wang J, Domingues O, et al. Targeting glioblastoma with $\mathrm{NK}$ cells and $\mathrm{mAb}$ against NG2/CSPG4 prolongs animal survival. Oncotarget. 2013;4(9):1527-1546.

110. Graner MW, Raynes DA, Bigner DD, Guerriero V. Heat shock protein 70-binding protein 1 is highly expressed in high-grade gliomas, interacts with multiple heat shock protein 70 family members, and specifically binds brain tumor cell surfaces. Cancer Sci. 2009;100(10): 1870-1879.

111. Gercel-Taylor C, Atay S, Tullis RH, Kesimer M, Taylor DD. Nanoparticle analysis of circulating cell-derived vesicles in ovarian cancer patients. Anal Biochem. 2012;428(1):44-53.

112. Oosthuyzen W, Sime NE, Ivy JR, et al. Quantification of human urinary exosomes by nanoparticle tracking analysis. J Physiol. 2013;591(Pt 23): 5833-5842.
113. Kuate S, Cinatl J, Doerr HW, Uberla K. Exosomal vaccines containing the $\mathrm{S}$ protein of the SARS coronavirus induce high levels of neutralizing antibodies. Virology. 2007;362(1):26-37.

114. Rountree RB, Mandl SJ, Nachtwey JM, et al. Exosome targeting of tumor antigens expressed by cancer vaccines can improve antigen immunogenicity and therapeutic efficacy. Cancer Res. 2011;71(15): 5235-5244.

115. Battke C, Ruiss R, Welsch U, et al. Tumour exosomes inhibit binding of tumour-reactive antibodies to tumour cells and reduce ADCC. Cancer Immunol Immunother. 2011;60(5):639-648.

116. Blanc L, Barres C, Bette-Bobillo P, Vidal M. Reticulocyte-secreted exosomes bind natural IgM antibodies: involvement of a ROSactivatable endosomal phospholipase iPLA2. Blood. 2007;110(9): 3407-3416.

117. Mathivanan S, Simpson RJ. ExoCarta: A compendium of exosomal proteins and RNA. Proteomics. 2009;9(21):4997-5000.

118. Ronquist KG, Ek B, Stavreus-Evers A, Larsson A, Ronquist G. Human prostasomes express glycolytic enzymes with capacity for ATP production. Am J Physiol Endocrinol Metab. 2013;304(6): E576-E582.

119. O'Brien K, Rani S, Corcoran C, et al. Exosomes from triple-negative breast cancer cells can transfer phenotypic traits representing their cells of origin to secondary cells. Eur J Cancer. 2013;49(8):1845-1859.

120. Alcazar O, Hawkridge AM, Collier TS, et al. Proteomics characterization of cell membrane blebs in human retinal pigment epithelium cells. Mol Cell Proteomics. 2009;8(10):2201-2211.

121. Biasutto L, Chiechi A, Couch R, Liotta LA, Espina V. Retinal pigment epithelium (RPE) exosomes contain signaling phosphoproteins affected by oxidative stress. Exp Cell Res. 2013;319(13):2113-2123.

122. Svensson KJ, Christianson HC, Wittrup A, et al. Exosome uptake depends on ERK1/2-heat shock protein 27 signaling and lipid Raftmediated endocytosis negatively regulated by caveolin-1.J Biol Chem . 2013;288(24):17713-17724.

123. Graner MW. The unfolded protein response in glioblastomas: passing the stress test. CNS Oncology. 2013;2(6):469-472. Available from: http://www.futuremedicine.com/toc/cns/2/6. Accessed January 10, 2014.

124. Stahl PD, Barbieri MA. Multivesicular bodies and multivesicular endosomes: the "ins and outs" of endosomal traffic. Sci STKE. 2002;2002(141):pe32.

125. Skriner K, Adolph K, Jungblut PR, Burmester GR. Association of citrullinated proteins with synovial exosomes. Arthritis Rheum. 2006;54(12):3809-3814.

126. Shen B, Wu N, Yang JM, Gould SJ. Protein targeting to exosomes/ microvesicles by plasma membrane anchors. J Biol Chem. 2011;286(16):14383-14395.
Pharmacogenomics and Personalized Medicine

\section{Publish your work in this journal}

Pharmacogenomics and Personalized Medicine is an international, peerreviewed, open access journal characterizing the influence of genotype on pharmacology leading to the development of personalized treatment programs and individualized drug selection for improved safety, efficacy and sustainability. This journal is indexed on the American Chemical

\section{Dovepress}

Society's Chemical Abstracts Service (CAS). The manuscript management system is completely online and includes a very quick and fair peer-review system, which is all easy to use. Visit http://www.dovepress. com/testimonials.php to read real quotes from published authors. 